Original Paper http://ajol.info/index.php/ijbcs http://indexmedicus.afro.who.int

\title{
Diversité floristique, structure et distribution des groupements végétaux des parcours d'accueil des transhumants dans la Basse et Moyenne Vallée de l'Ouémé au Bénin
}

\author{
Sylvanus Hanania Sèton HONVOU ${ }^{*}$, Boya André $\mathrm{ABOH}^{1,2}$, Clément SEWADE ${ }^{3}$, \\ Oscar TEKA ${ }^{1}$, Bernard Christophe GANDONOU ${ }^{4}$, Madjidou OUMOROU ${ }^{5}$ et Brice SINSIN ${ }^{1}$
}
${ }^{1}$ Laboratoire d'Écologie Appliquée, Faculté des Sciences Agronomiques/Université d'Abomey-Calavi 01 BP 526 Cotonou, Bénin.
${ }^{2}$ Laboratoire des Sciences Animales et Halieutiques, Université Nationale d'Agriculture, République du Bénin (West Africa), BP 90 Kétou, République du Bénin.
${ }^{3}$ Unité de Recherche en Foresterie et Conservation des Bio-ressources, Laboratoire des Sciences Végétales, Horticoles et Forestières, Université Nationale d'Agriculture, BP 43 Kétou, Bénin.
${ }^{4}$ Laboratoire de Physiologie Végétale et d'Étude des Stress Environnementaux, Faculté des Sciences et Techniques, Université d'Abomey-Calavi, 01BP 526 Tri postal, Cotonou, République du Bénin.
${ }^{5}$ Unité de Recherche-Phytosociologie, Espaces Protégés et Pastoraux, Agro-écosystèmes, conservation et valorisation des espèces endogènes (UR-PEPPAC), Laboratoire de Recherche en Biologie Appliquée (LARBA). Université d'Abomey-Calavi, 01BP 526 Tri postal, Cotonou, République du Bénin.
*Auteur correspondant ; E-mail: sylvanos01@gmail.com; Tel.: (00 229) 97053098

\author{
Received: 26-09-2020 \\ Accepted: 18-02-2021 \\ Published: 28-02-2021
}

\section{RESUME}

La perte de la biodiversité végétale, liée à la dégradation des parcours est l'un des défis pour les pays d'accueil des transhumants. La présente étude visait à analyser les caractéristiques structurales et la diversité floristique des groupements végétaux dans la Basse et Moyenne Vallée de l'Ouémé (BMVO). Les données phytosociologiques, collectées dans 113 relevés selon la méthode de Braun-Blanquet (1932), ont été soumises à une DCA avec le logiciel R 3.3.2. Ainsi, 173 espèces regroupées dans 127 genres et 45 familles ont été recensées. Au total, 4 groupements végétaux ont été identifiés : les groupements GI à Andropogon gayanus var squamulatus et Mitragyna inermis; GII à Paspalum notatum et Aeollanthus pubescens; GIII à Panicum maximum et Calopogonium mucunoides et le groupement végétal GIV à Tridax procumbens et Daniellia oliveri. La diversité floristique est liée à la topographie, la nature du sol et son humidité, et l'intensité d'exploitation des terres. Les thérophytes et les phanérophytes ont été les plus abondants dans tous les groupements. Par contre, les hémicryptophytes ont été plus dominants dans le groupement GI. Les espèces à large distribution et à distribution continentale ont été plus abondantes et dominantes dans tous les groupements. Cette recherche servira de base pour l'analyse de la dynamique de la végétation des terres de parcours de la BMVO.

(C) 2021 International Formulae Group. All rights reserved.

Mots clés : Parcours, facteurs écologiques, types biologiques, types phytogéographiques, Bénin. 


\title{
Floristic diversity, structure and distribution of plant communities of rangelands of the transhumant in the Lower and Middle Oueme Valley in Benin
}

\begin{abstract}
The loss of plant biodiversity as a sign of the degradation of rangelands is one of the major challenge for transhumant host countries. The aim of this study was to analyze the structural characteristics and the floristic diversity of plant communities in the Lower and Middle Oueme Valley (BMVO). Phytosociological data collected in 113 plots using the Braun-Blanquet method (1932), were submitted to DCA under the software R.3.3.2. About, 173 species belonging to 127 genera and 45 families were inventoried. A total of 4 plant communities have been identified: GI of Andropogon gayanus var squamulatus and Mitragyna inermis; GII of Paspalum notatum and Aeollanthus pubescens; GIII of Panicum maximum and Calopogonium mucunoides and GIV of Tridax procumbens and Daniellia oliveri. The nature of the soil and its humidity, the topography and the land use intensity were decisive for plant communities'distribution and for the flora diversity. The therophytes and phanerophytes were the most abundant in all the plant communities. In contrast, hemicryptophytes were more dominant in the floodplain clusters. Species with large distribution and continental distribution were more abundant and dominant in all the plant communities. This study will be the base line data to analyze the dynamic of vegetation of BMVO rangeland.
\end{abstract}

(C) 2021 International Formulae Group. All rights reserved.

Keywords: Rangelands, ecological factors, life form, chorological type, Benin.

\section{INTRODUCTION}

La conservation de la biodiversité est, de nos jours, un impératif pour toute société s'inscrivant dans l'optique du développement durable. La connaissance de la diversité végétale des espèces utilisées par les populations s'avère nécessaire pour mener des actions adéquates (Sèwadé, 2017). Ainsi, le développement de l'élevage extensif des herbivores domestiques au Bénin comme ailleurs en Afrique au Sud du Sahara nécessite une bonne connaissance de sa diversité végétale. En effet, les animaux de ce système d'élevage s'alimentent essentiellement du fourrage constitué de la végétation spontanée des parcours naturels et des résidus de récoltes. Pour ce mode d'élevage, la transhumance est une stratégie d'adaptation aux aléas climatiques adoptée par la majeure partie des éleveurs. Elle consiste en une exploitation opportuniste des ressources pastorales durant les crises fourragères saisonnières afin que les troupeaux tirent profit de la diversité écologique et de la complémentarité entre les différentes zones agro-climatiques du pays (Kiema et al., 2014). Malheureusement, la forme et les manifestations de la transhumance sont de nos jours bouleversées et soldées par des violences entrainnant des cas de mort d'homme aussi bien dans le rang des éleveurs que dans celui des agriculteurs. Ainsi, les pasteurs sont obligés d'investir de nouveaux territoires dans des zones plus clémentes et qui, dans un passé récent, étaient encore peu utilisées et bien pourvues en ressources fourragères (Sawadogo et al., 2012 ; Kiema et al., 2014). Dès lors, la transhumance et l'agriculture peuvent avoir un impact à même de modifier l'évolution de la succession végétale et d'entraîner des modifications du milieu des fois irréversiblement (Dan et al., 2012 ; Diallo et al., 2013).

La zone de la Base et Moyenne Vallée de l'Ouémé (BMVO) du Bénin, constitue depuis les années 1980, un milieu privilégié de destination finale des flux des animaux transhumants nationaux (TN) en provenance du nord Bénin et de ceux des animaux transhumants transfrontaliers (TTF) en provenance des pays voisins, notamment le Nigéria, le Niger, le Burkina Faso, le Mali et la Mauritanie (Ange et al., 2014 ; Honvou et al., 2018). Elle dispose des potentiels élevés de ressources en eau constitués par le fleuve 
Ouémé et ses affluents et de ressources végétales peu ou pas caractérisées (Ali et al., 2014 ; Obossou et al., 2020).

Selon Diallo et al. (2013), la transhumance non contrôlée est une menace de dégradation des graminées pérennes. Par ailleurs, les activités anthropiques sont considérées tantôt comme des facteurs favorables à l'augmentation de la biodiversité, tantôt comme des facteurs d'homogénéisation de la flore et des paysages (Botoni et al., 2006). Selon Toko Imorou (2013), la topographie est aussi l'un des principaux facteurs déterminants la diversité des communautés végétales à l'échelle stationnaire dans des conditions climatiques homogènes. L'écosystème de la BMVO connait depuis quelques années des perturbations diverses liées aux pressions anthropiques et aux effets de la variation climatique. Malheureusement, les études effectuées sur la diversité floristique ont porté seulement sur les forêts sacrée ou communautaires qui sont moins sollicitées par les éleveurs (Ali et al., 2014 ; Obossou et al., 2020). Par ailleurs, ces études n'ont pas particulièrement tenu compte de la topographie et intégré les stations récentes d'accueil des transhumants. Ainsi, l'objectif de cette étude est de (i) déterminer les groupements végétaux de la BMVO et (ii) d'analyser les caractéristiques structurales et la diversité floristique en relation avec la topographie, et l'emprise anthropique. L'hypothèse de recherche sous-tendant cette étude se base sur le fait que les groupements végétaux se discriminent suivant la topographie et le degré d'exploitation des terres d'une part et d'autre part ces groupements sont dominés par des graminées vivaces et des espèces de l'élémentbase Guinéo congolaise.

\section{MATERIEL ET METHODES \\ Zone d'étude}

L'étude a été menée dans la Basse et Moyenne Vallée de l'Ouémé (BMVO) (Figure 1), située entre $2^{\circ} 00$ et $2^{\circ} 45$ de longitude Est et $6^{\circ} 34$ et $7^{\circ} 32$ de latitude Nord au Sud Est du Bénin soit une superficie de $3722 \mathrm{~km}^{2}$. La BMVO correspond dans la partie Sud à la zone chorologique guinéo congolaise et dans la partie Nord à la zone chorologique guinéo soudanienne (Adomou et al., 2006). Elle correspond aux districts phytogéographiques de Pobè, du Plateau, de la Vallée de l'Ouémé et du Zou dans sa partie nord (Adomou, 2010). Les différentes formations sont constituées des espèces ligneuses telles que Mitragyna inermis, Lonchocarpus sericeus, Pterocarpus santalinoides, Morinda lucida et Kigelia africana (Honvou et al., 2018 ; Obossou et al., 2020). Le climat du site d'étude est de type subéquatorial dans la basse vallée et guinéosoudanienne dans la moyenne vallée (Adomou, 2010). Il est caractérisé par un régime pluviométrique bimodal dans la basse vallée et un régime à tendance unimodale dans la moyenne vallée. La précipitation moyenne annuelle sur la période de 1960 à 2017 est estimée à $1321,3 \mathrm{~mm}$ à la station de Cotonou et $1124,08 \mathrm{~mm}$ à la station de Bohicon (DNM, 2018). Les températures moyennes annuelles enregistrées sont de $27,99^{\circ} \mathrm{C}$ à Bohicon et de $27,6{ }^{\circ} \mathrm{C}$ à Cotonou sur la période de 1968 à 2017 (DNM, 2018). Le réseau hydrographique de la zone d'étude est composé du fleuve Ouémé et de son principal affluent, le fleuve Zou. Les observations ont montré que la crue du fleuve Ouémé s'amorce en juillet et atteint son niveau maximal en septembre et son retrait débute en octobre.

\section{Choix des sites de relevés phytosociologiques}

Une enquête a été réalisée dans la BMVO auprès de la population pour repérer le passage des troupeaux transhumants. Une prospection de la BMVO a été effectuée à l'aide de la carte d'occupation des terres réalisée à partir de l'image LANDSTAT ETM 2014 afin d'identifier les différentes unités d'occupations des terres. $\mathrm{Au}$ total, 38 sites/villages ayant accueilli les éleveurs transhumants pendant au moins 8 années, de 2007 à 2017 ont été retenus. Les placeaux de relevés d'inventaires floristiques ont été installés suivant la variation de l'unité topographique et les types de formations végétales. 


\section{Evaluation phytoécologique des aires de pâturage}

L'évaluation phytoécologique des pâturages a consisté à inventorier la flore du terroir de la BMVO. Au total, 113 relevés phytosociologiques ont été réalisés dans des placeaux de $10 \mathrm{~m} \times 10 \mathrm{~m}$ pour la strate herbacée la plus homogène possible. Ces relevés phytosociologiques ont été réalisés en octobre et décembre en 2017 suivant la méthode de Braun-Blanquet (1932). Ces mois correspondent aussi à la phase de maturation des espèces végétales. Les données enregistrées concernant la végétation ont porté sur la topographie, le type de formation végétale, le recouvrement, la liste des espèces et les coefficients d'abondance-dominance suivant l'échelle de Braun-Blanquet (1932). Les espèces végétales recensées ont été herborisées pour leur identification à l'Herbier National du Bénin. La nomenclature utilisée est celle de Hutchinson et Dalziel (1954-1972) et de Lebrun et Storck (1991-1997). L'ordination des relevés et les plans factoriels ont été réalisés par le logiciel R.3.3.2 avec le package vegan sur la base d'une DCA. Le package labdsv a permis de calculer les valeurs indicatrices des espèces caractéristiques des différents groupements végétaux identifiés.

\section{Caractérisation des groupements végétaux}

L'analyse de la diversité spécifique de la végétation de la zone d'étude a consisté à caractériser les différents groupements végétaux. Pour mettre en relation les assemblages des espèces avec les conditions environnementales et les perturbations, l'analyse a pris en compte aussi bien les descripteurs taxinomiques (indices) que non taxinomiques tels que les traits biologiques, phytogéographiques.

Les descripteurs taxinomiques utilisés ont porté sur la richesse spécifique (S) dans chaque groupement, l'indice de Shannon (H'), l'indice de l'équitabilité de Piélou (E) et l'indice de similitude de Jaccard (Ij).

La richesse spécifique (S) est le nombre total d'espèces dans un groupement. L'indice de diversité spécifique de Shannon H' est défini selon la formule suivante :

$$
\mathrm{H}^{\prime}=-\sum \mathrm{Pi} \log _{2} \mathrm{PiPi}=\mathrm{Rmi} / \mathrm{RM} \text { total, }
$$
où : Rmi est le recouvrement moyen de l'espèce i dans les relevés du groupement végétal et RM total est le recouvrement moyen de toutes les espèces des relevés du groupement végétal.

H' s'exprime en bits. Il varie généralement entre 1 et 5 bits. $\left(H^{\prime}>3,5\right)$ signifie une forte diversité au sein du groupement végétal alors les conditions de la station sont très favorables à un grand nombre d'espèces dans des proportions quasi-égales. $\mathrm{H}^{\prime}$ faible $\left(\mathrm{H}^{\prime}<2,6\right)$ signifie que les conditions $\mathrm{du}$ milieu sont très défavorables et induisent une forte spécialisation des espèces.

Le niveau de diversité atteint au niveau de chaque groupement végétal a été mesuré au moyen de l'indice d'équitabilité de Pielou E :

$\mathrm{E}=\mathrm{H}^{\prime} / \log _{2} \mathrm{SAvec} \mathrm{H}^{\prime}=$ indice de diversité spécifique de Shannon du groupement végétal et $\mathrm{S}=$ richesse spécifique totale. E est compris entre 0 et 1 . L'équitabilité de Pielou est faible si $\mathrm{E}$ est compris entre 0 0,6 ; moyen si $\mathrm{E}>0,6$ et élevé si $\mathrm{E}>0,8$. Si E tend vers 0 alors la quasi-totalité des individus appartiennent à une seule espèce et quand $\mathrm{E}$ prend la valeur 1 alors toutes les espèces ont exactement le même recouvrement.

La similitude entre les groupements végétaux a été étudiée par l'indice de similitude de Jaccard (Ij) (Jaccard, 1901). Cet indice est défini selon la formule suivante :

$$
\mathrm{Ij}=\mathrm{c} /(\mathrm{a}+\mathrm{b}-\mathrm{c}) \text { Avec } \mathrm{c}=\text { nombre }
$$
d'espèces communes aux groupements $\mathrm{G} 1$ et $\mathrm{G} 2, \mathrm{a}=$ nombre d'espèces de $\mathrm{G} 1$ et $\mathrm{b}=$ nombre d'espèces de G2.Cet indice Ij varie de 0 à 1 . Si $\mathrm{Ij}>0,5$ les milieux sont similaires et $\mathrm{si} \mathrm{Ij}<0,5$ il y a dissimilitude entre les milieux.

Les types biologiques liés au modèle phénologique distingués, sont ceux définis d'après la classification de Raunkiaer (1934) et modifié par Lebrun (1947). Cette classification regroupe : les Phanérophytes $(\mathrm{Ph})$, Chaméphytes (Ch), Géophytes (Ge), Hémicryptophytes (He), Thérophytes (Th). Les types phytogéographiques adoptés correspondent aux grandes subdivisions chorologiques de Lebrun (1947) modifiées par White (1983) et admises pour l'Afrique. Il s'agit des espèces à large distribution 
comprenant : les cosmopolites (Cos) ; pantropicales (Pan) ; paléotropicales (Pal); afro-américaines (AA). Les espèces à distribution continentale comprenant : afrotropicales (AT); afro-malgaches (AM) ; les plurirégionales africaines (PA), les soudanozambézienne (SZ), et les soudano-guinéen (SG). Les espèces de l'élément base comprennent : les guinéo-congolaises (GC) et les soudaniennes (S). Pour tenir compte du poids des espèces pour un caractère, la comparaison a été faite par l'analyse des spectres bruts (SB) et les spectres pondérés
(SP) des types biologiques, des types phytogéographiques. Le spectre brut est donné par la formule suivante :

SB

$$
\begin{aligned}
& =\frac{\text { Effectif du trait considéré }}{\text { Effectif total de tous les traits identifiés }} \\
& \times 100
\end{aligned}
$$

Le spectre pondéré est donné par la formule suivante :

SP

$=\frac{\sum \text { recouvrement moyen du trait considéré }}{\sum \text { recouvrement de tous les traits identifiés }}$ $\times 100$

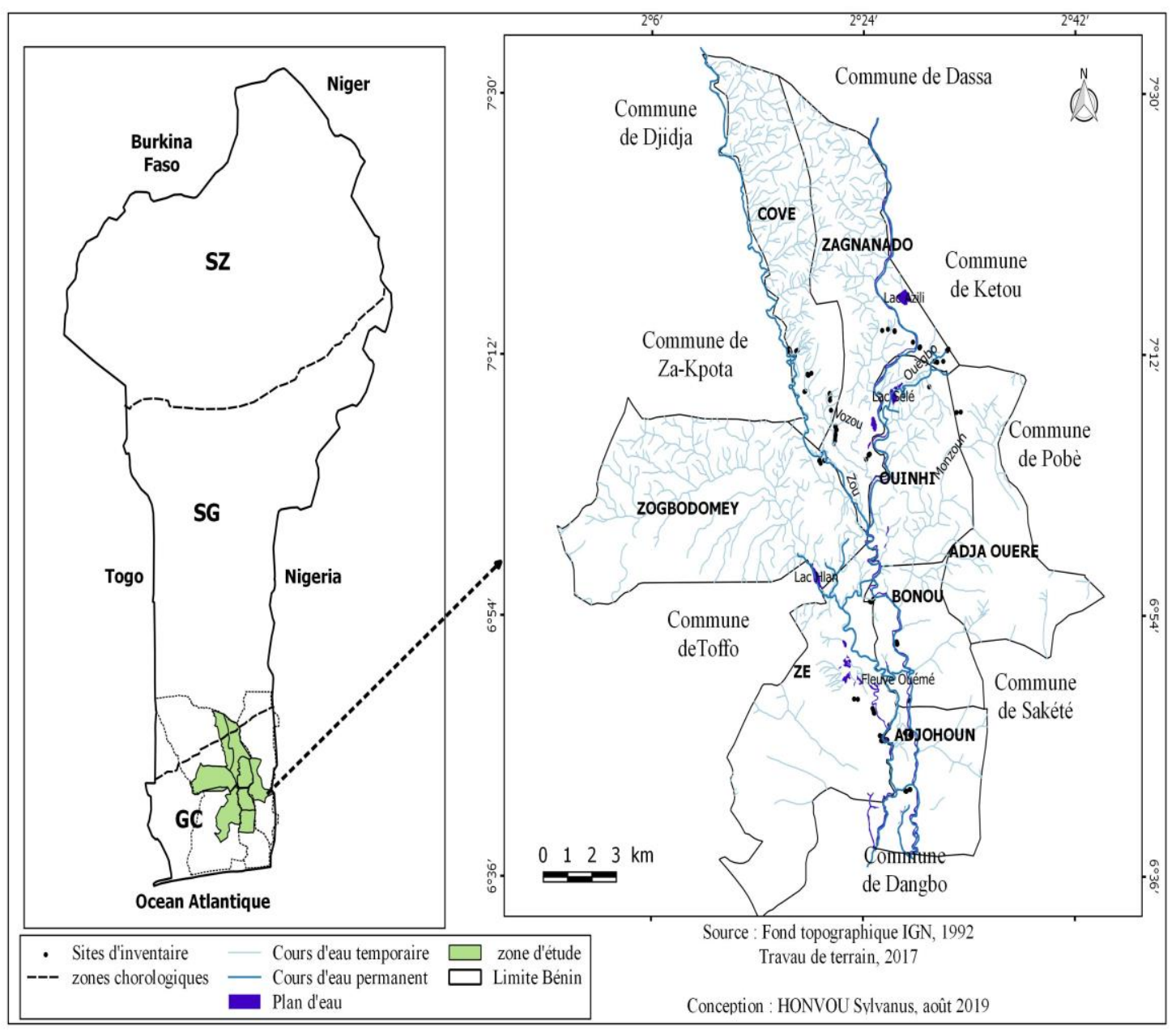

Figure 1 : Carte du Bénin montrant la zone d'étude et les sites inventaires. 


\section{RESULTATS}

\section{Typologie et caractéristiques des groupements végétaux}

Au total, 173 espèces regroupées en 45 familles et 127 genres ont été recensées. La DCA a montré sur la matrice des 113 relevés croisés avec les 173 espèces que les deux premiers axes contribuent à $56,96 \%$ à l'explication des variabilités observées (Tableau 1). Ce tableau indique les résumés des valeurs propres et les pourcentages de variance correspondante. Le plan factoriel a révélé une discrimination des relevés suivant les axes 1 et 2 (Figure 2). L'axe 1 montre dans sa partie négative, les relevés réalisés dans la plaine alluviale d'inondation saisonnière (espace plane où les cours d'eau ne s'encaissent pas, mais qui est submergée de façon saisonnière par la crue).. Par contre, la partie positive de l'axe des abscisses, a indiqué les relevés réalisés sur le milieu et le haut du versant. L'axe 2 révèle, dans sa partie positive, une concentration des relevés effectués dans les savanes herbeuses et savanes-arborées. La partie négative de l'axe montre une concentration de relevés réalisés dans les champs et jachères.

Le résultat de l'analyse globale de la végétation a permis le découpage de quatre groupements végétaux GI, GII, GIII et GIV au sein des formations végétales étudiées. Le groupement GI est composé de 53 relevés réalisés dans les savanes herbeuses et savanes arborées sur un sol à texture argileuse. Le groupement GII est composé de 16 relevés réalisés dans les jachères sur un sol argilolimoneuse. Le groupement GIII est composé de 25 relevés réalisés dans les jachères sur un sol à texture sablo-limoneuse. Le groupement GIV est composé de 19 relevés issus des champs sur un sol à texture sablo-limoneuse.

L'analyse statistique a révélé les espèces indicatrices de chaque groupement (Tableau 2). En combinant ces résultats avec les espèces dominantes, il est ressorti que :

- le groupement végétal GI est celui à Andropogon gayanus var squamulatus et Mitragyna inermis des savanes herbeuses et arborées, de la plaine alluviale d'inondation saisonnière sur un sol à texture argileux;

- le groupement végétal GII, est un groupement à Paspalum notatum et Aeollanthus pubescens des jachères du bas du versant de faible inondation saisonnière sur un sol à texture argilo-limoneux;

- le groupement végétal GIII est celui à Panicum maximum et Calopogonium mucunoides des jachères au milieu de versant sur un sol à texture sablolimoneux ;

- le groupement végétal GIV correspond à celui à Tridax procumbens et Daniellia oliveri des champs du haut de versant sur un sol à texture sablo-limoneux.

\section{Diversité des différents groupements végétaux}

Les espèces recensées sont repartis entre 70 et 112 espèces par groupement. La plus forte richesse spécifique est obtenue dans le groupement GI à $A$. gayanus var squamulatus et $M$. inermis des savanes herbeuses et arborées de la plaine alluviale et la plus faible dans le groupement GIV à $T$. procumbens et $D$. oliveri des champs du haut du versant (Tableau 3).

L'indice de diversité de Shannon (H') a varié de 3,35 à 4,39 bits et l'équitabilité de Pielou de 0,55 à 0,67 (Tableau 3). L'indice de Shannon est élevé au niveau du groupement GIII, GII et GI. L'équitabilité de Pielou a été moyen pour les groupements GII et GIII et faible pour le groupement GI. Par contre l'indice de Shannon est moyen au niveau du groupement GIV avec une équitabilité faible.

Le nombre de familles au sein des groupements a varié de 28 à 36. Les Poaceae (14 à 17 espèces), les Fabaceae ( 9 à 13 espèces), les Convolvulaceae (1 à 6 espèces), les Cyperaceae ( 2 à 6 espèces) et les Asteraceae (4 à 5 espèces) étaient les cinq familles ayant plus de richesse spécifique.

\section{Similitude entre les groupements végétaux}

L'indice de similitude de Jaccard entre les différents groupements végétaux est compris entre 0,23 et 0,45 (Tableau 4). Cependant, les groupements GI et GII ont été 
floristiquement proches $(0,44)$. De même, les groupements GII et GIII ont été floristique proche $(0,45)$. Par contre, la similitude floristique a été faible entre les groupements GI et GIV $(0,23)$, entre les groupements GII et $\operatorname{GIV}(0,30)$.

\section{Spectres des types biologiques}

Les thérophytes suivis des phanérophytes ont été les plus représentés dans tous les groupements végétaux (Figure 3a). Par contre, les proportions des chaméphytes, hémicryptophytes et géophytes étaient moyennes. Les hémicryptophytes ont été plus dominantes dans les groupements GI et $(45,36 \%)$ et GII $(50,57 \%)$. Les chaméphytes $(28,83 \%)$ ont été dominantes dans le groupement GIII (Figure 3b). Par contre, les thérophytes $(56,14 \%)$ ont été dominantes dans le groupement GIV.

\section{Spectres des types phytogéographiques}

Les résultats ont révélé que les espèces à large distribution sont plus représentées (47,83 à 62,50\%) et les plus dominantes $(49,31$ à 76,96\%) dans tous les groupements végétaux (Figure 4a). Elles sont suivies des espèces à distribution continentale en termes d'abondance $(28,75$ à $35,87 \%)$ dans tous les groupements. En termes de dominance elles sont suivies des espèces de l'élément base Guinéo-congolaise $(22,19 \%)$ dans le groupement GII et des espèces à distribution continentale dans les autres groupements (Figure 4b). La proportion des espèces de l'élément base Guinéo-congolaise ont été plus élevés dans les groupements GIII et GIV que dans ceux des GI et GII. La proportion des espèces de l'élément base Guinéo-congolaise est plus élevé que celle des espèces soudaniennes dans tous les groupements. Seul le groupement végétal GII a enregistré la présence des espèces soudaniennes.

Tableau 1 : Valeurs propres et pourcentage d'inertie des quatre premiers axes.

\begin{tabular}{lcccc}
\hline Axes & Axe 1 & Axe 2 & Axe 3 & Axe 4 \\
\hline Valeurs propres & 0,86 & 0,72 & 0,59 & 0,60 \\
Pourcentage d'inertie & 30,93 & 26,03 & 21,29 & 21,76 \\
Cumul des pourcentages & 30,93 & 56,96 & 78,24 & 100 \\
\hline
\end{tabular}

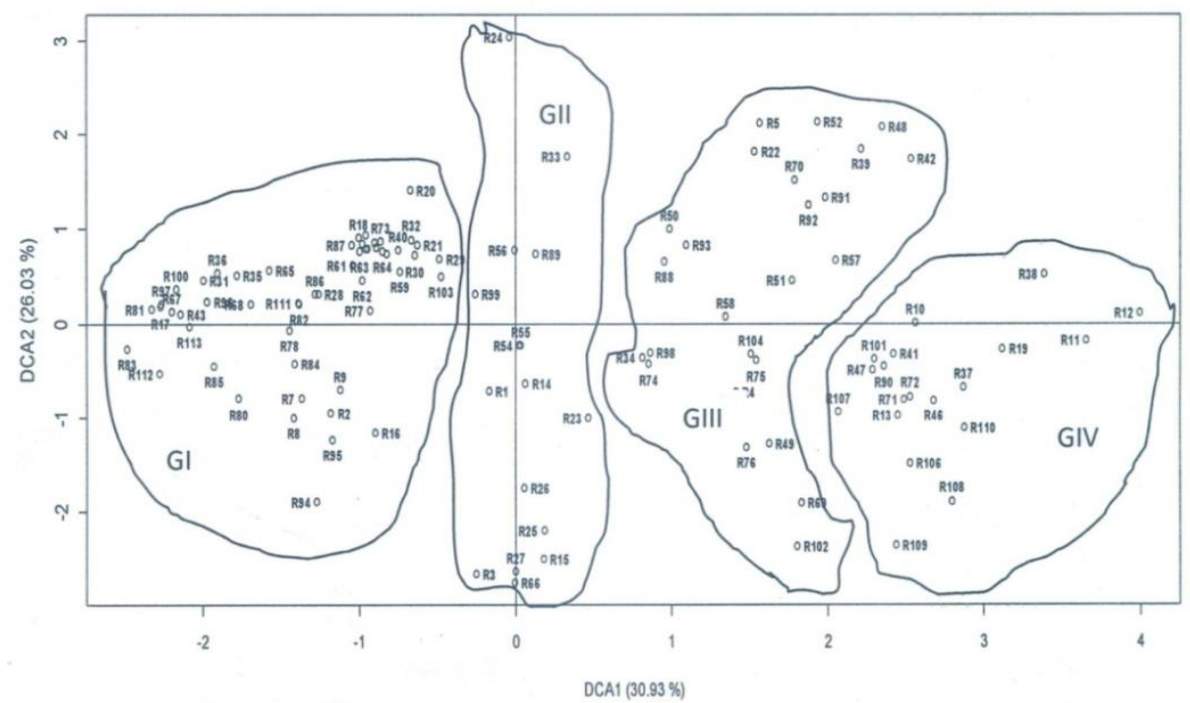

Figure 2 : Présentation de la projection sur les axes 1 et 2 du plan factoriel de la DCA appliquée aux 113 relevés. 
Tableau 2 : Valeurs indicatrices des espèces caractéristiques des groupements végétaux.

\begin{tabular}{|c|c|c|c|}
\hline $\begin{array}{l}\text { Groupements } \\
\text { (écosystème) }\end{array}$ & Espèces & $\begin{array}{c}\text { Valeurs } \\
\text { indicatrices }(\%)\end{array}$ & Probabilité \\
\hline \multirow{13}{*}{$\begin{array}{l}\text { Groupement I } \\
\text { (Plaine alluviale } \\
\text { d'inondation } \\
\text { saisonnière) }\end{array}$} & Paspalum scrobiculatum $L$. & 68,29 & 0,001 \\
\hline & Andropogon gayanus var & 64,82 & 0,001 \\
\hline & squamulatus (Hochst.) Stapf & & \\
\hline & Ipomoea aquatica Forssk. & 56,27 & 0,001 \\
\hline & $\begin{array}{l}\text { Mitragyna inermis (Willd.) } O . \\
\text { Ktze. }\end{array}$ & 55,07 & 0,001 \\
\hline & Leersia hexandra $S w$ & 41,35 & 0,001 \\
\hline & Ludwigia decurrens Walt. & 32,15 & 0,02 \\
\hline & Mimosa pigra Linn. & 30,19 & 0,001 \\
\hline & $\begin{array}{l}\text { Cissampelos mucronata A. } \\
\text { Rich. }\end{array}$ & 27,66 & 0,023 \\
\hline & Melochia corchorifolia $L$. & 21,00 & 0,025 \\
\hline & Crinum zeylanicum L. & 19,24 & 0,029 \\
\hline & Pterocarpus santalinoides $D C$. & 18,95 & 0,013 \\
\hline & Celosia laxa Shum et Thonn & 15,09 & 0,038 \\
\hline \multirow{9}{*}{$\begin{array}{l}\text { Groupement II } \\
\text { (Bas du versant de } \\
\text { faible inondation } \\
\text { saisonnière) }\end{array}$} & Passiflora foetida $L$. & 58,00 & 0,004 \\
\hline & $\begin{array}{ll}\text { Sorghum } & \text { arundinaceum } \\
\text { (Willd.) Stapf } & \end{array}$ & 56,54 & 0,001 \\
\hline & Aeollanthus pubescens Benth. & 43,43 & 0,001 \\
\hline & Corchorus fascicularis Lam. & 37,40 & 0,008 \\
\hline & Paspalum notatum Flugge & 36,50 & 0,001 \\
\hline & Aeschynomene indica $L$. & 28,94 & 0,02 \\
\hline & Ludwigia abyssinica A. Rich. & 26,06 & 0,004 \\
\hline & Hyptis spicigera Lam. & 23,87 & 0,005 \\
\hline & Physalis angulata $L$ & 23,38 & 0,047 \\
\hline \multirow{10}{*}{$\begin{array}{l}\text { Groupement III } \\
\text { (Milieu du } \\
\text { versant) }\end{array}$} & Panicum maximum Jacq. & 50,71 & 0,001 \\
\hline & Centrosema pubescens Benth. & 41,77 & 0,01 \\
\hline & $\begin{array}{l}\text { Calopogonium mucunoides } \\
\text { Desv }\end{array}$ & 37,55 & 0,002 \\
\hline & $\begin{array}{l}\text { Chromolaena odorata (Linn.) } \\
R \text {. King et H. Robinson }\end{array}$ & 36,92 & 0,003 \\
\hline & Triumfetta rhomboidea Jacq. & 31,11 & 0,003 \\
\hline & Mucuna pruriens (Linn.) DC. & 27,35 & 0,002 \\
\hline & $\begin{array}{l}\text { Rottboellia cochinchinensis } \\
\text { (Lour.) W.D. Clayton }\end{array}$ & 25,00 & 0,04 \\
\hline & $\begin{array}{l}\text { Pennisetum polystachion (L.) } \\
\text { Schult. }\end{array}$ & 20,02 & 0,039 \\
\hline & Triclisia subcordata Oliv. & 19,61 & 0,013 \\
\hline & Hibiscus asper Hook. $f$. & 15,20 & 0,022 \\
\hline
\end{tabular}




\begin{tabular}{|c|c|c|c|}
\hline & $\begin{array}{l}\text { Andropogon tectorum Schum. } \\
\text { \& Thonn. }\end{array}$ & 12,00 & 0,02 \\
\hline \multirow{12}{*}{$\begin{array}{l}\text { Groupement IV } \\
\text { (Haut du versant) }\end{array}$} & Tridax procumbens $L$ & 76,46 & 0,001 \\
\hline & $\begin{array}{l}\text { Daniellia oliveri (Rolfe) Hutch. } \\
\& \text { Dalz. A }\end{array}$ & 36,84 & 0,001 \\
\hline & Hyparrhenia rufa (Nees) Stapf. & 26,25 & 0,001 \\
\hline & Cynodon dactylon (L.) Pers. & 25,57 & 0,002 \\
\hline & Oldenlandia corymbosa Linn. & 20,25 & 0,002 \\
\hline & Brachiaria sp & 15,79 & 0,007 \\
\hline & $\begin{array}{l}\text { Crotalaria leprieurii Guill. et } \\
\text { Perr. }\end{array}$ & 15,79 & 0,008 \\
\hline & $\begin{array}{l}\text { Rhynchelytrum repens (Willd.) } \\
\text { C.E. Hubbard }\end{array}$ & 15,79 & 0,009 \\
\hline & Eragrostis tremula Steud. & 10,53 & 0,041 \\
\hline & $\begin{array}{l}\text { Indigofera stenophylla Guill. \& } \\
\text { Perr. }\end{array}$ & 10,53 & 0,045 \\
\hline & $\begin{array}{l}\text { Parkia biglobosa (Jacq.) R. Br. } \\
\text { ex Benth. }\end{array}$ & 10,53 & 0,05 \\
\hline & Pterocarpus erinaceus Poir & 10,53 & 0,043 \\
\hline
\end{tabular}

Tableau 3 : Richesse spécifique (S), indices de diversité de Shannon $(\mathrm{H})$, indice d'équitabilité de Piélou (E) et nombre de familles (F) des groupements végétaux.

\section{\begin{tabular}{lllllll}
\hline Groupements végétaux & Texture du sol & Formations végétales & $\mathbf{S}$ & $\mathbf{F}$ & $\mathbf{H}$ & $\mathbf{E}$
\end{tabular} (écosystème)}

\begin{tabular}{|c|c|c|c|c|c|c|}
\hline GI : Andropogon gayanus & Argileux & Savanes herbeuses et & 11 & 36 & 3,81 & 0,56 \\
\hline $\begin{array}{l}\text { var squamulatus et } \\
\text { Mitragyna inermis (plaine } \\
\text { alluviale } \\
\text { d'inondation saisonnière) }\end{array}$ & & arborées & 2 & & & \\
\hline $\begin{array}{l}\text { GII : Paspalum notatum et } \\
\text { Aeollanthus pubescens } \\
\text { (bas du versant de faible } \\
\text { inondation saisonnière) }\end{array}$ & $\begin{array}{l}\text { Argilo- } \\
\text { limoneuse }\end{array}$ & Jachère & 80 & 31 & 4,19 & 0,66 \\
\hline $\begin{array}{l}\text { GIII : Panicum maximum } \\
\text { et Calopogonium } \\
\text { mucunoides (Milieu du } \\
\text { versant) : }\end{array}$ & Sablo-limoneuse & Jachère & 92 & 33 & 4,39 & 0,67 \\
\hline $\begin{array}{l}\text { GIV : Tridax procumbens } \\
\text { et Daniellia oliveri (Haut } \\
\text { du versant) }\end{array}$ & Sablo-limoneuse & Champs & 70 & 27 & 3,35 & 0,55 \\
\hline
\end{tabular}


Tableau 4 : Indice de similitude de Jaccard entre les différents groupements végétaux.

\begin{tabular}{lcccc}
\hline Groupements & GI & GII & GIII & GIV \\
\hline GI & & & & \\
GII & 0,44 & & & \\
GIII & 0,37 & 0,45 & & \\
GIV & 0,23 & 0,30 & 0,36 & \\
\hline
\end{tabular}
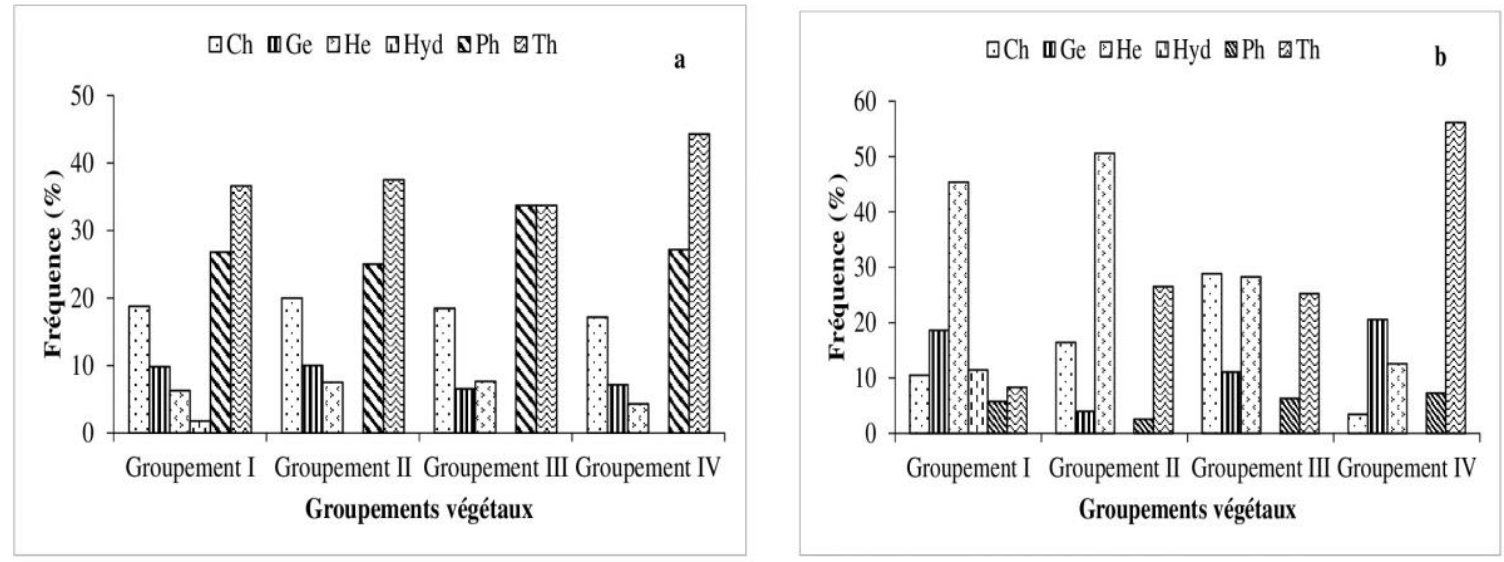

Th=Thérophytes ; He=Hémicryptophytes ; Ge=Géophytes $; \mathrm{Ch}=$ Chamephytes $; \mathrm{Ph}=$ Phanérophytes; Hyd= Hydrophytes

Figure 3: Spectre brut (a) et pondéré (b) des types biologiques des groupements végétaux.
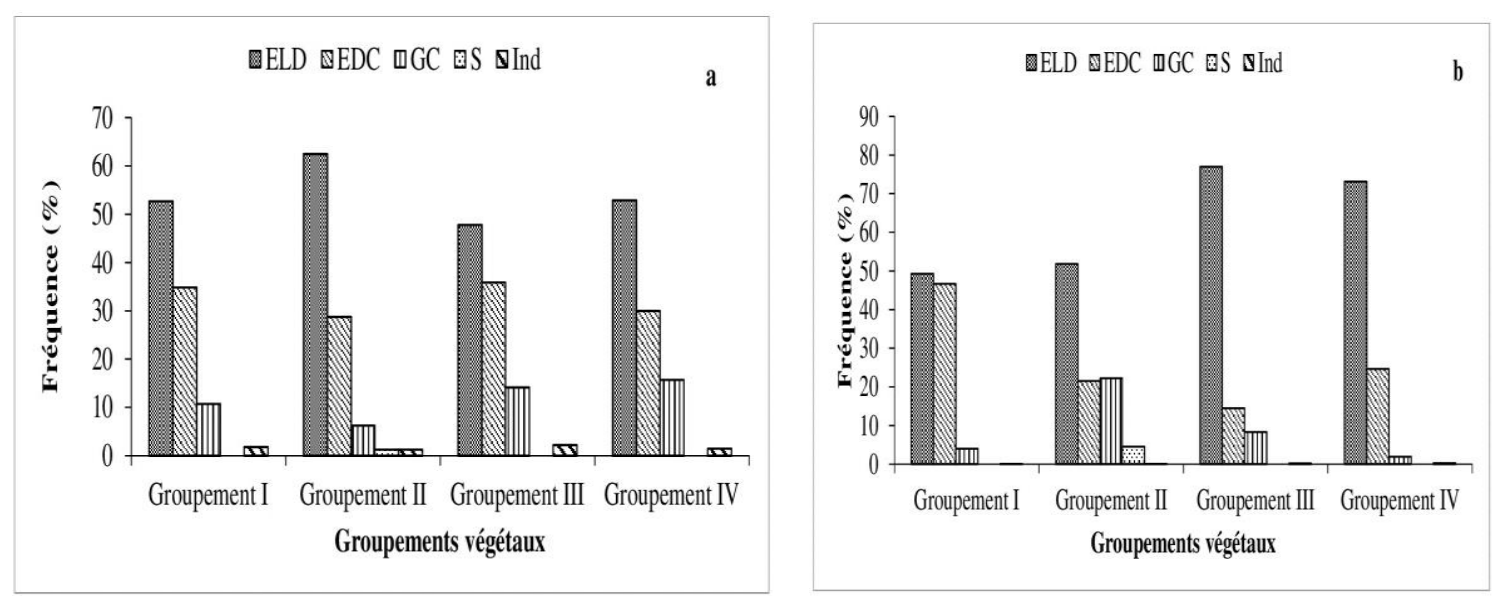

ELD=espèces à large distribution; $\mathrm{EDC}=$ espèces à distribution continentale ; GC=espèces de l'élément base Guinéocongolaise; $\mathrm{S}=$ espèces de l'élément base Soudanienne ; Ind=Indéterminé

Figure 4 : Spectre brut (a) et pondéré (b) des types phytogéographiques des groupements végétaux. 


\section{DISCUSSION}

\section{Diversité floristique et répartition écologique des groupements végétaux}

La DCA réalisée sur la matrice des 113 relevés et 173 espèces a révélé la discrimination de quatre types de groupements végétaux distincts. Cette richesse spécifique des herbacées représente $6,16 \%$ de la flore du Bénin estimée à 2807 espèces (Akouègninou et al., 2006).

Les facteurs déterminants de cette distribution spatiale des groupements végétaux sont les gradients environnementaux tels que, d'une part la topographie, la nature du sol et l'humidité; et d'autre part l'intensité d'exploitation des terres. Chaque groupement végétal est caractérisé par ses conditions édaphiques spécifiques et les espèces indicatrices qui contribuent fortement au maintien du groupement malgré les perturbations. Selon Jordan et al. (2006), l'extinction ou la fluctuation importante de l'abondance des espèces indicatrices peuvent affecter sérieusement d'autres espèces et conduire voire accélérer l'extinction de la communauté végétale entière. Par ailleurs, les espèces indicatrices peuvent être utilisées pour analyser les tendances de la végétation et leurs variables environnementales sous-jacentes (Diallo et al., 2013). Aussi, l'influence significative des facteurs édaphiques et du relief dans la répartition spatiale des groupements végétaux au sein du paysage naturel, avait-elle également été mise en évidence par d'autres études (Nduwimana et al., 2015). Notre étude révèle que dans la BMVO, la répartition des groupements est déterminée par les facteurs topographiques et édaphiques. En effet, le groupement végétal GI à A. gayanus var squamulatus et $M$. inermis des savanes herbeuses et savanes arborées des plaines alluviales à inondation saisonnière est présente sur un sol à texture argileuse. Le groupement végétal GII à $P$. notatum et $A$. pubescens des jachères situées en bas du versant à faible inondation saisonnière est sur un sol à texture argilo-limoneuse. En milieu du versant, s'est implanté le groupement végétal GIII à $P$. maximum et $C$. mucunoides des jachères sur un sol à texture sablo-limoneuse et en haut du versant et le groupement végétal GIV à $T$. procumbens et $D$. oliveri des champs est sur un sol à texture sablo- limoneuse.

Les facteurs édaphiques ne sont pas les seuls qui déterminent la répartition des groupements végétaux. En effet, sur des sols de même texture, la DCA différencie deux formations végétales juxtaposées en fonction de l'intensité d'exploitation des terres telles que les jachères et les champs de cultures d'une part et les savanes herbeuses et savanes arborées d'autre part. L'influence du gradient anthropique dans la répartition des groupements végétaux des parcours pastoraux est mise en évidence dans la zone soudano guinéenne (Toko Imorou, 2013), au Nord du Bénin (Assani et al., 2017) et dans la zone guinéenne du Togo (Amegnaglo et al., 2018).

Il en résulte que la zone d'étude est caractérisée par des conditions très hétérogènes en raison des différences et de la dynamique des types de végétation (mosaïque de savane herbeuse, savane arborée, jachère et champs), et de la composition floristique révélée à travers les 173 espèces recensées. Lesse et al. (2016) et Assani et al. (2017) ont trouvés des valeurs similaires respectivement 171 et 178 espèces dans les zones parcourues par les transhumants au nord Bénin. Cependant, cette richesse floristique des terres de parcours des transhumants de la BMVO reste inférieure aux 199 espèces obtenues par Aboh et al. (2009) dans la zone de transition soudano guinéenne du Bénin. Cette différence floristique serait liée aux pressions anthropiques et à l'écosystème. La pression humaine quasi permanente à travers l'agriculture et l'élevage dans les formations végétales sont les facteurs de diminution de la richesse floristique (Dan et al., 2012). Selon Adomou et al. (2006), plusieurs facteurs peuvent expliquer ces différences, dont notamment les variations des conditions pédoclimatiques notamment leurs influences sur la distribution spatiale et géographique des flores. Dans ce milieu d'étude, la prédominance des Poaceae, Fabaceae, Convolvulaceae et Asteraceae corrobore les résultats d'autres auteurs pour la flore guinéocongolaise (Kouassi et al., 2010) et dans la flore du Bénin (Akoègninou et al., 2006). 
La richesse floristique, a été relativement plus importante dans le groupement GI de la plaine alluviale à inondation saisonnière que dans les autres groupements. Les conditions édaphiques du milieu pourraient expliquer cette différence. En effet, les sols de ce groupement GI bénéficient chaque année de l'apport en alluvions de la crue (Etene et al., 2016 ; Yabi et al., 2018), de la persistance de l'humidité du sol, même en période sèche à cause de la texture du sol qui retient de l'eau. Ces conditions édaphiques sont plus que favorables au développement de plusieurs plantes. Toko Imorou (2013) a montré que le niveau d'humidité du sol dans les formations végétales crée un microclimat qui favorise l'installation et le développement de nombreuses espèces. Ainsi, la forte valeur de l'indice de Shannon dans le groupement GI est la résultante de ces conditions pédoclimatiques. L'indice de régularité de Piélou obtenu est faible dans le groupement GI et traduit ainsi la dominance de certaines espèces telles que A. gayanus var. squamulatus et E. pyramidalis. L'indice de diversité de Shannon et l'indice de Piélou sont élevés pour le GII des jachères du bas versant de faible inondation saisonnière. Cela indiquerait une bonne diversité et une équirépartition des individus au sein du groupement. Au niveau du groupement GIII des jachères du milieu de versant sur sol à texture sablo-limoneuse, l'indice de diversité de Shannon est élevé et l'indice de régularité de Piélou moyen. Ce qui signifierait que le milieu est diversifié avec une répartition égale du recouvrement des espèces. Par contre, ces indices ont été plus faibles pour les champs du haut de versant. Cela montrerait que l'abondance est irrégulièrement distribuée entre beaucoup d'espèces végétales dans ce milieu où certaines espèces comme $T$. procumbens ont été dominantes dans le groupement. Ces espèces pionnières sont souvent abondantes dans les milieux mis à nus.

La richesse floristique a eu tendance à diminuer avec l'augmentation de la pression anthropique. Cette tendance pourrait être liée à la faible résilience de nombreuses espèces face à l'intensité de la perturbation causée par la pâture et les activités agricoles. Elle pourrait être également liée à une forte compétition entre les espèces pour les ressources nutritives. En revanche, la tendance inverse a été observée pour les formations peu perturbées. Toutefois, Zampaligre et al. (2019) ont montré que de nombreuses espèces s'installent à la faveur de la perturbation induite par la pâture. Ainsi, l'action anthropique et, en particulier, l'impact de la pâture sur la biodiversité végétale sont assez controversés (Achard et al., 2001). En effet, ils sont tantôt considérés comme un facteur favorable à l'augmentation de la biodiversité, tantôt comme un facteur d'homogénéisation de la flore et des paysages (Botoni et al., 2006).

\section{Analyse de la diversité floristique et intérêt des groupements pour les ruminants}

Pour les formes biologiques, le milieu de la plaine alluviale et du bas du versant qui reçoivent l'inondation saisonnière a été caractérisée par la prédominance des hémicryptophytes notamment $P$. scrobiculatum L., A. gayanus var. squamulatus (Hochst.) Stapf., $\quad$ S. arundinaceum (Willd.) Stapf, et des géophytes telles que I. aquatica Forssk. et Cyperus rotundus $L$.qui sont des espèces adaptées à ce milieu. D'autres auteurs (Kidjo et al., 2011 ; Sieban et al., 2016) ont rapporté la présence de ces espèces dans des milieux analogues. Par contre, les thérophytes ont été les plus abondantes dans les groupements végétaux GIV et GIII. Cette prédominance des thérophytes au détriment des hémicryptophytes indiquerait une perturbation du milieu de la zone d'étude, une dégradation des pâturages comme les travaux d'autres auteurs l'ont montré (Diallo et al., 2013). La prédominance des thérophytes au niveau des groupements sur sol à texture sablo-limoneuse pourrait en partie s'expliquer par la consommation excessive des graminées par le bétail. Ce qui a favorisé le développement des espèces les moins consommées ou refusées comme les thérophytes au détriment des graminées pérennes et des légumineuses qui sont les plus appétées. Par ailleurs, le piétinement et l'arrachage des souches des herbacées, aussi 
bien par les sabots que par les dents des animaux, entrainent par endroits des plages de sol nu qui sont colonisées par des espèces invasives comme Chromolaena odorata (Aboh et al., 2008). Ce serait probablement le cas du groupement GIII où l'espèce $C$. odorata a été identifiée comme espèce caractéristique. La prédominance des thérophytes dans le groupement GIV et des chaméphytes dans le groupement GIII plus que dans les groupements GI et GII serait liée à une intensité élevée de la pratique agricole et de la pâture. Par conséquent, les types biologiques reflètent à la fois les paramètres structuraux et les conditions pédoclimatiques variées d'une végétation.

La physionomie des groupements végétaux étudiés a été dominée par les espèces à large distribution pantropicale, paléotropicale et plurirégionale. Cette prédominance des espèces à large distribution dans les groupements GI et GII est surtout liée à l'humidité du sol (Toko Imorou, 2008). Par ailleurs la dominance de ces espèces dans les groupements GIII et GIV traduit un degré d'altération des pâturages de la zone dû aux pratiques pastorales et agricoles (Sinsin et Oumorou, 2000). La dominance des espèces à large distribution et des espèces à distribution continentale suivi du faible recouvrement des espèces de l'élément base dans les groupements végétaux, traduirait l'appartenance de la zone d'étude au domaine Guinéo-Congolaise perturbé. Cette perturbation de la flore originelle GuinéoCongolaise par l'apparition des espèces soudanienne serait liée aux pratiques de la transhumance associée aux variations de changement climatique.

Au-delà des herbacées utilisées dans l'alimentation du bétail, les éleveurs ont recours à certains ligneux fourragers comme aliment d'appoint de leur troupeau en saison sèche où les herbacées ne sont plus disponibles (Sèwadé et al., 2018 ; Honvou et al., 2018). Les espèces souvent citées par ces auteurs sont Khaya senegalensis, Afzeliaafricana, Prosopis africana, Pterocarpus erinaceus, M. inermis, Lonchocarpus sericeus Pterocarpus santalinoides et D. olivera. Dès lors, l'alimentation des troupeaux aussi bien des communautés locales que ceux des transhumants, repose intégralement sur la cueillette des ligneux fourragers des aires de parcours, sans efforts d'aménagement et de renouvellement soutenu. De plus, la végétation naturelle est de plus en plus menacée par d'autres facteurs tels que l'urbanisation, l'agriculture, la carbonisation et l'exploitation (Sèwadé, 2017). A cet effet, une prise de conscience serait nécessaire au niveau de tous les acteurs utilisant les ressources des terres de parcours pour définir des stratégies de gestions rationnelles de ces ressources au regard du rôle important joué dans la survie des troupeaux aussi bien des transhumants nationaux que régionaux.

\section{Conclusion}

Cette étude réalisée sur les terres de parcours d'accueil des transhumants de la BMVO s'intègre dans la problématique globale des services rendus par la biodiversité à l'agriculture. Elle a permis d'identifier quatre groupements végétaux dans cette zone hétérogène. L'étude a révélé qu'en plus de la topographie et de la structure du sol, le gradient anthropique surtout le mode d'exploitation et de gestion de la végétation a une influence sur la variation de la structure et de la diversité floristique. La richesse floristique est plus élevée dans la savane herbeuse et savane arborée de la plaine alluviale à d'inondation saisonnière avec une dominance des hémicryptophytes qui constituent la ressource alimentaire recherchée par les ruminants. Toutefois, ces groupements sont menacés par une dégradation qui se manifeste par une régression progressive des graminées pérennes et leur remplacement par des espèces annuelles et les plantes envahissantes dont $C$. odorata; une diminution des espèces de l'Elément Base au détriment des espèces à large distribution géographique. Les résultats de cette étude suggèrent de modifier les modes d'exploitation de l'écosystème afin de permettre à la flore de se reconstituer pour revenir à son état stable et de procéder à son aménagement compte tenu du degré de dégradation. A cet effet, un plan 
d'aménagement pourrait être préalablement élaboré à la suite de cette étude sur l'occupation, la productivité fourragère et la valeur pastorale des terres de la BMVO. Tout ceci permettra de définir une stratégie de l'utilisation rationnelle des ressources des terres de parcours de cette région.

\section{CONFLIT D'INTERETS}

Les auteurs déclarent qu'ils n'ont aucun conflit d'intérêts dans le cadre du présent manuscrit.

\section{CONTRIBUTIONS DES AUTEURS}

Les auteurs ci-dessous ont participé de diverses manières à la conception de cet article. SHSH a collecté, traité et analysé les données puis rédigé et corrigé le présent manuscrit; $\mathrm{ABA}$ et OT sont intervenus dans la conception du dispositif, le traitement et l'analyse des données ; la correction du présent manuscrit ; $\mathrm{CS}, \mathrm{CBG}, \mathrm{MO}$ et $\mathrm{BS}$ ont contribué à la lecture et la correction du présent manuscrit.

\section{REFERENCES}

Aboh BA, Oumorou M, Houinato M, Sinsin B. 2009. Analyse biologique et phytogéographique des savanes colonisées par Chromolaena odorata et Hyptis suaveolens dans la région de Bétécoucou (Bénin). Syst. Geogr. Pl., 79: 81-92. DOI: 10.2307/20649773.

Aboh BA, Houinato M, Oumorou M, Sinsin B. 2008. Capacités envahissantes de deux espèces exotiques, Chromolaena odorata (asteraceae) et Hyptis suaveolens (lamiaceae), en relation avec l'exploitation des terres de la région de bétécoucou (Bénin), Belgian Journal of. Botany, 141(2): 113-128. DOI: 10.2307/20794660

Achard F, Hiernaux P, Banoin M. 2001. Les jachères naturelles et améliorées en Afrique de l'Ouest. In De la Jachère Naturelle à la Jachère Améliorée. Le Point des Connaissances, Montrouge, Floret C, Pontanier R (éds). John Libbey Eurotext : France ; 201-239.

Adomou AC, Sinsin B, van der Maesen LJG. 2006. Phytosociological and chorological approaches to phytogeography: a study at meso-scale in Benin. Systematics and Geography of Plants, 76: 155-178. www.jstor.org/stable/20649708

Adomou AC. 2010. Territoires phytogéographiques au Bénin. In Atlas de la biodiversité de l'Afrique de l'Ouest (Tome 1), Sinsin B, Kampman D (éds). BIOTA: Cotonou, Bénin et Frankfurt/Maim, Allemagne ; 134-143.

Akoègninou A, Van Der Burg WJ, Van Der Maesen LJG. 2006. Flore Analytique $d u$ Bénin. Backhuys Publishers : Netherlands ; 1033 p.

Ali RKFM, Odjoubere J, Tente ABH, Sinsin AB. 2014. Caractérisation floristique et analyse des formes de pression sur les forêts sacrées ou communautaires de la Basse Vallée de l'Ouémé au Sud-Est du Bénin. Afrique SCIENCE, 10 (2) : 243 257

Amegnaglo KB, Dourma M, Akpavi S, Wala K, Diwediga B, Atakpama W, Agbodan KML, Batawila K, Akpagana K. 2018. Caractérisation des formations végétales pâturées de la zone guinéenne du Togo: typologie, évaluation de la biomasse, diversité, valeur fourragère et régénération. International Journal of Biological and Chemical Sciences, 12(5): 2065-2084.

Ange M, Bekpa K, Sinsin B. 2014. Transhumance and conflicts management on Agonlin plateau in Zou department (Benin). Journal of Biodiversity and Environmental Sciences, 4 (5): 132-145.

Assani AS, Alkoiret IT, Worogo HS, Agbayigbo AA, Houinato M. 2017. Floristic Characterization of the Natural Rangelands of the Classified Forest of Upper Alibori in Northern Benin. Saudi Journal of Engineering and Technology, 2: $\quad 380-389$. DOI: 10.21276/sjeat.2017.2.10.4.

Botoni EL, Daget P, Cesar J. 2006. Activités de pâturage, biodiversité et végétation pastorale dans la zone Ouest du Burkina Faso. Revue d'Elevage et de Médecine Vétérinaire des Pays Tropicaux, 59(1-4) : 31-38. 
Braun-Blanquet J. 1932. Plant sociology: The study of plant communities, (Translated by G.D. Fuller and H.S. Conard.). McGraw-Hill: New York and London.

Dan CBS, Sinsin BA, Mensah GA, Lejoly J. 2012. Influence des activités anthropiques sur la diversité floristique des communautés végétales de la forêt marécageuse de Lokoli au Sud-Bénin. Int. J. Biol. Chem. Sci., 6 (6): 3064-3081. DOI:

http://dx.doi.org/10.4314/ijbcs.v6i6.8

Diallo H, El Hadji Faye EH, Kone B, Bindelle J, Lejoly J, Maiga M. 2013. Biodiversité et valeur pastorale des herbacées de la Réserve de Fina (Mali). In African Plant Diversity, Systematics and Sustainable Development - N. Beau, S. Dessein \& E. Robbrecht (eds), Proceedings of the XIXth AETFAT Congress, held at Antananarivo, Madagascar, 26-30 April 2010. Scripta Botanica Belgica, 50:111120.

Direction Nationale de la Météorologie (DNM). 2018. Données climatiques journalières de température et de pluviométrie dans les stations de Cotonou et Bohicon de1978 à 2017.

Duvigneaud P 1980. La synthèse écologique. Doin. Paris, 380p.

Etene CG, Arouna O, Babadjide C, Kodja JD. 2016. Pluies maximales et dégradation des terres à Bonou dans le bassin de l'Ouémé au Bénin. Revue semestrielle de Géographie du Bénin, 20 : 134-151.

Jaccard P. 1901. Lois de distribution de la flore alpine dans le bassin de Dranses et quelques régions voisines. Bulletin de la Société Vaudoise des Sciences Naturelles, 37: 241-272.

Jordan F, Liu WC, Davis AJ. 2006. Topological keystone species: measures of positional importance in food webs. Oikos, 112: 535-546. https://www.jstor.org/stable/3548719.

Kidjo FC, Djossa BA, Houngbedji MG, Lougbegnon T, Codjia JTC, Sinsin B. 2011. Ecologie alimentaire du Sitatunga (Tragelaphusspeckei, Sclater, 1864) dans les sites Ramsar du Sud-Bénin.
International Journal of Biological and Chemical Sciences, 5(2): 603-617. DOI: http://dx.doi.org/10.4314/ijbcs.v5i2.72126.

Kiema A, Tontibomma GB, Zampaligré N. 2014. Transhumance et gestion des ressources naturelles au Sahel : contraintes et perspectives face aux mutations des systèmes de productions pastorales. VertigO, la revue électronique en sciences de l'environnement [En ligne], 14(3). URL: http://vertigo.revues.org/15404; consulté le 26 juin 2017. DOI : 10.4000/vertigo.15404.

Kouassi AF, Adou YCY, Ipou. IJ, Kamanzi K. 2010. Diversité floristique des zones côtières pâturées de la Côte d'Ivoire : cas $\mathrm{du}$ cordon littoral Port-Bouët GrandBassam (Abidjan). Sciences \& Nature, 7(1) : 69-86. DOI: 10.4314/scinat.v7i1.59936.

Lebrun J. 1947. La végétation de la plaine alluviale au Sud du lac Edouard. Institut des Parcs Nationaux du Congo Belge, Exploration du Parc National Albert, Mission Lebrun (1937-1938). Fascicule 1 : 472-800.

Nduwimana A, Riera B, Bizuru E. 2015. Influence des facteurs écologiques sur la composition et la diversité des unités de végétation du paysage de la Malagarazi (Burundi). Revue Écologie. (Terre et Vie). 70(3) : 213-230.

Obossou FK, Adjakpa JB, Dansi MF, Dissou FE, Azonningbo SHW, Yedomonhan H. 2020. Étude floristique et structurale de la forêt communautaire d'ahouanhouzoukan à tévèdji dans la commune de Ouinhi au sud-bénin. Rev. Ivoir. Sci. Technol., 35 : $288-308$.

Oumorou M. 2003. Etudes écologique, floristique, phytogéographique et phytosociologiques des inselbergs du Bénin. Thèse de doctorat, Université Libre de Bruxelles, $210 \mathrm{p}$.

Raunkiaer C. 1934. The Life's Forms of Plants and Statistical Plant Geography. Oxford University Press: London; 632 p.

Sawadogo I, Devineau JL, Fournier A. 2012. État des ressources pastorales dans une 
terre d'accueil et de transit des pasteurs transhumants : le terroir de Kotchari (sudest du Burkina Faso). Rev. Écol. (Terre Vie), $67:$ 157-178.

Sèwadé C. 2017. Diversité, biomasse foliaire des ligneux fourragers et capacité de charge des terres de parcours des zones de transition Guinéo-Congolaise/ Soudanienne du Bénin. Thèse de Doctorat. FSA/UAC, 242 p.

Sèwadé C, Lokonon EB, Azihou AF, Akouèhou SG, Mensah GA, GlèlèKakaï LR, Houinato M. 2018. Use diversity and farmer's preference of 48 local fodder trees: a comparative analysis of three sociolinguistic groups from the GuineoCongolese / Sudanian transition zone of Benin. Annales des Sciences Agronomiques, 22 (1) : 33-52.

Sieben EJJ, Collins NB, Corry FTJ, Kotze DC, Job N, Muasya AM, Venter CE, Mtshali H, Zondo SA, Janks M, Pretorius L. 2014. The vegetation of grass lawn wetlands of floodplains and pans in semi-arid regions of South Africa: Description, classification and explanatory environmental factors. South African Journal of Botany, 104: 215-224. DOI: http://dx.doi.org/10.1016/j.sajb.2015.11. 003.

Sinsin B, Oumorou M. 2000. Etude de la diversité spécifique du groupement à Cochlospermum tinctorium A. Rich. des savanes arbustives du nord-Benin. Acta Bot. Gallica, 147(4) : 345-360. DOI : https://doi.org/10.1080/12538078.2000.1 0515866

Toko Imorou I. 2013. Effets des facteurs abiotiques sur la répartition spatiale des groupements végétaux dans la zone de transition soudano-guinéenne du Bénin. Int. J. Biol. Chem. Sci., 7(6): 2178-2192. DOI:

http://dx.doi.org/10.4314/ijbcs.v7i6.1

Toko I. 2008. Etude de la variabilité spatiale de la biomasse herbacée, de la phénologie et de la structure de la végétation le long des toposéquences du bassin supérieur du fleuve Ouémé au Bénin. Thèse de doctorat, Université d'Abomey-Calavi, Abomey-Calavi, p. 241.

White F. 1983. The vegetation of Africa. A descriptive memoir to accompany the Unesco/Aetfat/Unso vegetation map of Africa. UNESCO, Natural Resources Research, 20: 356p.

Yabi I, Yolou I, Yerima LO, Koudjegan E, Afouda F. 2018. Valorisation agricole des vallées de la commune de Ouinhi (SudBénin) : entre atouts et contraintes. $\mathrm{La}$ Revue des Sciences Sociales « Kafoudal » $1: 11-33$.

Zampaligre N, Kagambega WF, Sanou L, Sawadogo L. 2019. Impact of Grazing Intensity on Floristic Diversity and Woody Structure in Grazing Area Near Kaboré Tambi National Park (Burkina Faso). Journal of Agriculture and Environmental Sciences, 8(2): 106-115. DOI: 10.15640/jaes.v8n2a13. 